\title{
Renal Cell Carcinoma: A Case Series with Integrative Treatment
}

\author{
Miranda Costa*, Elena Panutich and Heather Zwickey
}

National College of Natural Medicine, Helfgott Research Institute, 2220 SW 1st Ave, Portland, OR 97201, USA

\begin{abstract}
Objective: Renal cell carcinoma (RCC) is a rare and difficult to treat cancer. The case series aimed to observe the effects of medicinal mushrooms and integrative oncology care for renal cell carcinoma.

Clinical Features: Patients diagnosed with RCC who were being treated with surgery and chemotherapy and were administered integrative treatment in the form of supplements and medicinal mushrooms and/or combinations of mushrooms.

Interventions and outcomes: Integrative care included a combination of supplements and the medicinal mushrooms Ganoderma lucidum, Trametes versicolor, Cordyceps sinensis, Grifola frondosa, and Lentinus edodes in combination or alone, which was initiated following adjuvant care. These patients experienced increased overall survival compared to standard adjuvant treatment alone.

Conclusion: Beneficial effects of integrative care were observed in this case series. Disease progression and symptoms related to adjuvant treatment were significantly decreased in these patients after integrative supportive care was initiated and effects persisted through the final observation period. Further controlled studies are needed among larger groups of patients to determine the clinical efficacy of medicinal mushrooms and integrative oncology in the treatment of RCC.
\end{abstract}

Keywords: Integrative medicine research; Integrative oncology; Renal cell carcinoma; Kidney cancer

\section{Introduction to Renal Cell Carcinoma}

Current data estimates there will be 61,560 new cases of Kidney and Renal Pelvis Cancer this year, and 14,000 people will die of the disease in the United States [1]. At presentation many patients are asymptomatic, until the disease is advanced. Nearly $30 \%$ of patients have metastatic disease at the time of diagnosis of renal cell carcinoma $[2,3]$. Currently, only $5 \%$ of new cases present with the classic Virchow triad involving flank pain, hematuria, and abdominal mass, which is associated with poor prognosis. Combining standard treatments for RCC with integrative care may improve overall survival, promote immune surveillance of cancer cells, promote apoptosis, and inhibit angiogenesis.

Clinically, there are three main renal neoplasm subtypes: clear cell RCC (80-90\%), papillary RCC, and chromophobe RCC. Each subtype determines cancer-specific survival prognoses. The 5-year overall survival for all RCC subtypes is $72.4 \%$, which is an improvement compared to previous survival rates, which is largely attributed to targeted therapies [1]. Metastatic disease usually involves the lung, lymph nodes, bone, liver, and brain [4]. The 5-year overall survival for regional RCC is $64.6 \%$, whereas distant, and/or metastatic, disease 5 -year overall survival is $12.1 \%$ [1].

Surgery can be curative in patients without metastatic disease, and is indicated for patients with stages I, II, and III localized disease [5]. Patients with stage I or II RCC with invasion of the urinary collecting system have a 5 year survival rate of $43 \%$ and 10 year-survival rate of $41 \%$ [6,7]. Patients with stage IV have identified renal capsular invasion, collecting system invasion, and microvascular invasion are approximately 3.3 times greater risk of having recurrence of disease after resection compared to those that do not have capsular invasion [8]. While diagnosis is frequently not made until disease is locally advanced or nonresectable, many patients who have surgical resection eventually experience disease recurrence. Stage IV median survival is 28 months in patients that are able to enroll in clinical trials [9].
Adjuvant treatment after nephrectomy currently has no established benefit in patients who have undergone a complete resection of local disease. The NCCN guidelines recommend follow-up imaging, or active surveillance, after treatment of localized surgical excision every 3 to 6 months. Chemotherapy, gemcitabine and cisplatin, do not have an established role in the management of patients with advanced RCC and is considered a category 3 -treatment option [10,11]. However, in patients that have metastatic disease to the bladder, the first line adjuvant treatment is gemcitabine and cisplatin according to NCCN guidelines. Metastatic disease to the bladder is rare, and prognosis is poor with a 3 -year survival rate of $20 \%$ with multiple metasteses [12]. The recommended treatment for patients that experience metastatic disease is a metastasectomy, with the exception of the brain and bone. Patients with metastases to the lung, and subsequent metastasectomy show a 5-year survival rate of $36-54 \%$ [13].

Large reviews on the active constituents and clinical applications of medicinal mushrooms have been growing in the past several years, and the active constituents have demonstrated to be useful as novel treatment options for cancers that evade the anti-growth signals, apoptosis, and angiogenesis [14-18]. Medicinal mushrooms that have been investigated and target these mechanisms of resistance include Ganoderma lucidum, Trametes versicolor, Cordyceps sinensis, Grifola frondosa, and Lentinus edodes.

*Corresponding author: Miranda Costa, National College of Natural Medicine, Helfgott Research Institute, 2220 SW 1st Ave, Portland, OR 97201, USA, Tel: 5417784130; E-mail: doctormirandacosta@gmail.com

Received December 10, 2015; Accepted January 04, 2016; Published January 15,2016

Citation: Costa M, Panutich E, Zwickey H (2016) Renal Cell Carcinoma: A Case Series with Integrative Treatment. J Integr Oncol S1: 002. doi:10.4172/2329-6771. S1-002

Copyright: ( 2016 Costa M, et al. This is an open-access article distributed under the terms of the Creative Commons Attribution License, which permits unrestricted use, distribution, and reproduction in any medium, provided the original author and source are credited. 
Ganoderma lucidum, also known as Reishi, has demonstrated antiangiogenic effects in human lung cancer cells and promoted apoptosis in these cells [19]. Reishi has also been shown to be cytotoxic in drugresistant small-cell lung cancer cells and alters gene expression in these carcinoma cells, similar to the effects of chemotherapeutic agents [20]. Extracts of Reishi can also inhibit cell growth and inhibit actin in human urethral and bladder cancer [21].

A systematic review and meta-analysis of Trametes versicolor used with chemotherapy demonstrates increased overall survival of cancer patients compared to patients completing standard of care alone [22]. This study highlights the safety of the medicinal mushroom, Trametes versicolor, used with a variety of chemotherapies in a variety of cancers where no notable adverse side-effects were observed in this systematic review.

Trametes versicolor, also known as Turkey tail, can promote antitumor effects by suppressing cell proliferation and increasing apoptosis in human leukemia cells [23]. Research on the extracts of turkey tail show the powerful effects of direct inhibition of transforming growth factor- $B$, which controls cell proliferation, differentiation, and apoptosis in cancer cells [24]. Combined with cisplatin this mushroom demonstrates enhanced anti-tumor and anti-metastatic activity in addition to reducing nephrotoxicity in mice models [25].

Extracted fractions from the medicinal mushroom Grifola frondosa (Maitake), of polysaccharides have been shown to inhibit cancer cell growth in a dose-dependent manner, and are selectively cytotoxic to cancer cells compared to non-cancer cells [26,27]. Extracts of G. frondosa have also exhibited potent activation of NK cells and prevention of metastatic disease by enhancing the effects of chemotherapy in mouse models [25].

Lentinus edodes, Shiitake mushroom, extracts has reduced tumor progression in bladder cancer patients undergoing chemotherapeutic agents, gemcitabine [28]. Also, high concentrations of shiitake mushroom extracts $(1-5 \mathrm{mg} / \mathrm{mL})$ have shown inhibition of tumor cell proliferation in carcinoma cells in vitro [29].

Chen et al. completed a review in 2013 of Cordyceps sinensis polysaccharides and the therapeutic benefits for the treatment of cancer [30]. Constituents, specifically cordycepin, extracted from this mushroom show growth inhibition and promote apoptosis in cancer cells [31]. Research in patients with lung cancer undergoing chemotherapy that incorporated cordyceps showed better tolerance to chemotherapy compared to standard chemotherapy alone [32]. Additionally, patients with lung cancer showed reduction in tumor size with continuous use of cordyceps compared to patients who did not incorporate cordyceps with standard chemotherapy [33].

Further investigation and examination of the mechanisms of the intrinsic and acquired resistance to standard care, and combining integrative care is urgently needed to improve the prognosis in RCC patients. This case series describes the clinical presentation of renal cell carcinoma in two male patients with metastatic disease. The cases highlight the use of medicinal mushrooms in the integrative management of renal cell cancer over four years following diagnosis and standard care. Overall survival and quality of life was improved in these patients with integrative care.

\section{A Case Series and Methods}

Physicians at local Integrative oncology centers and Naturopathic clinics were contacted for potential participants in the case series. Two patients diagnosed with RCC who were being treated with medicinal mushrooms and/or combinations of mushrooms were selected for the case series. Written consent from each patient was obtained for the inclusion in the case series. The clinical presentation, treatments, and follow-up data were obtained from the patient charts and directly from treating physicians. The quality of life, patient objectives, and survival is described in the case series.

\section{Case 1}

A 60-year-old male presented to his primary care doctor with hematuria, and weight loss. Diagnostic imaging and biopsy revealed renal cell carcinoma of the right kidney with metastatic spread to the bladder and ureters. The patient underwent surgical removal of his right kidney and eleven lymph nodes. Chemotherapeutic agents, Gemcitabine and Cisplatin, were initiated following surgery. After surgery and adjuvant chemotherapy the patient experienced side effects that included weight loss, poor appetite, constipation, myalgia, neuropathy and significant fatigue.

The patient presented to an integrative health center seeking supportive cancer care, immune support, vitality, and long-term robust health. At the initial visit the patient reported exercising daily, and utilizing self- prescribed supplemental vitamins, acupuncture, and hypnotherapy to maintain his health.

The patient was given an integrative plan to support his health and improve his quality of life while completing chemotherapy. The integrative plan included nutritional support, probiotics daily, melatonin $20 \mathrm{mg}$ daily, and a five mushroom formula 2 grams daily (Coriolus versicolor, Cordyceps sinensis, Grifola frondosa, Ganoderma lucidum, Lentinus edodes). Constitutional hydrotherapy was also recommended once every week. The patient was compliant with the initial treatment plan and subsequent reports revealed improved mood and tolerance of chemotherapy. Initially, chemotherapy had side effects of fatigue and anemia.

In the second year of treatment, follow up radiographic imaging revealed suspicious densities in bilateral lower lung fields. Video assisted thoracic surgery (VATS) was completed with patient reported increased pain and discomfort. Re-staging of the disease placed the patient at asymptomatic stage IV bladder cancer, with metastases to the lungs. Combination chemotherapy of methotrexate, vinblastine, adriamycin, and cisplatin was initiated one-month post resection. Neuropathy, anosmia, and thrombocytopenia were noted side effects following surgery and chemotherapy. Continued use of the five mushroom formulas during and after chemotherapy was emphasized for immune support following surgery and chemotherapy. Nutritional guidance emphasized eating green vegetables, whole grains, nuts, green tea, and avoiding sugar, artificial sweeteners, and other refined carbohydrates.

Three years following diagnosis of renal cell carcinoma the patient continued to exercise and remained hopeful with integrative care and felt positive about life. The Bacillus Calmette-Guerin (BCG), or tuberculosis vaccine, treatment was initiated to treat and prevent recurrence of bladder cancer. The patient reported subsequent bone pain and fatigue, and lab results revealed persistent anemia. Additional treatments were added to the patient's plan following radiographic imaging that revealed disease progression in the lungs. Treatment at the time included a drug trial for sutent (TKI), which resulted in gastrointestinal discomfort, flushing of the upper trunk, and fatigue. One month following drug trial patient reported depression, and continued gastrointestinal symptoms. Integrative care at this time 
incorporated L-glutamine, probiotics, curcumin, and licorice extract were added to the patient's treatment plan, which improved his gastrointestinal symptoms.

In the final year of this patient's life, four years following diagnosis, there was metastatic disease progression in the lung and eventually the brain. Stereotactic body radiation therapy to treat lung metastases, and whole brain radiation therapy was initiated. Following radiation therapies the patient reported profound fatigue and cognitive dissonance. The patient died a few months after initiating radiation therapies. This patient survived nearly 2 years beyond his expected prognosis.

\section{Case 2}

A 65 year-old-male presented to his primary care physician with gross hematuria. Past medical history was significant for hypertension, hyperlipidemia, and history of supraventricular tachycardia. Following CT imagining the patient underwent a radical nephrectomy. Pathology report described a multilobated tumor, consistent with clear renal cell carcinoma. Tumor size was $6.2 \mathrm{~cm}$ (2.4 inches) with no evidence of metastatic disease and a Furman nuclear grade III. The tumor was confined within the renal capsule, but extended into the renal sinus fat. Surgical margins were negative and there was no evidence of vascular invasion. Chest CT revealed two small nodules in the lungs with unknown significance. The patient's medications at diagnosis included lisinopril, simvastatin $40 \mathrm{mg}$ tablet, and aspirin $81 \mathrm{mg}$ daily. The patient's medical oncologist recommended active surveillance of his disease to include CT imaging of the chest and abdomen every three months for the next year. The patient initiated integrative naturopathic care, which included nutritional support and immune support; specifically Coriolus versicolor mushroom 3 grams daily, fish oil, alpha lipoic acid, hydrotherapy, and IV nutrient support.

One year following diagnosis the patient reported feeling well. A right-sided pulmonary nodule was visualized on routine chest radiograph. Several scattered pulmonary nodules of uncertain significance were present bilaterally. A right video-assisted thoracoscopic (VAT) surgery was performed and pathology revealed pulmonary metastases of the RCC cancer measuring $1.6 \mathrm{~cm}$. The surgical margins and resected lymph nodes were negative for malignancy. Following surgery the patient reported doing well; however, he had mild discomfort at the incision sites and a persistent dry cough. Integrative care at this time included IV vitamins, CoQ10 enzyme, Coriolus mushroom 3 grams daily, and L-carnitine with the recommendation to continue weekly hydrotherapy and to exercise daily. Laboratory results revealed elevated creatinine, and elevated ALT. All other labs were within normal limits.

Two years post nephrectomy and VAT surgery of right lung; disease pulmonary metastases were once again detected on CT. Imaging revealed subcentimeter pulmonary nodules and a subcentimeter liver nodule. PET scan was negative for increased FDG uptake. Despite disease progression this patient reported feeling generally well, and denied bony pain or difficulty breathing. Subsequent follow up imaging revealed no change in left kidney, and no lymphadenopathy. Pulmonary parenchyma and nodules seen previously were stable and showed no interval enlargement. The patient rotated use of mushrooms from Coriolus versicolor to a combination of Grifola frondosa, Ganoderma lucidum, and Lentinus edodes.

Radiologist report, three years following diagnosis, revealed increasing size of bilateral pulmonary nodules and right hilar adendopathy. The patient reported increasing anxiety following CT results, but continued to exercise daily and his energy levels remained elevated. The patient also stated having a great appetite and gaining 10 pounds, and denied any other symptoms related to disease progression. Integrative care plan described hydrotherapy, IV vitamins, vitamin $\mathrm{D}$, vitamin $\mathrm{C}$, vitamin $\mathrm{B}$ complex, mushroom combination powder (Grifola frondosa, Ganoderma lucidum, Lentinus edodes), L-carnitine, fish oil, alpha lipoic acid, and calcium/magnesium.

Evidence of systemic metastatic disease was detected four years later in the chest wall and lungs, and was determined to be nonresectable. The patient experienced hemoptysis and chronic cough and occasional nosebleeds. Medications at the time included aspirin $81 \mathrm{mg}$, simvastatin $40 \mathrm{mg}$, losartan, Lexapro $10 \mathrm{mg}$, Tessalon $100 \mathrm{mg}$, and singular $10 \mathrm{mg}$ daily. Integrative support included intravenous vitamins every other week, Coriolus versicolor mushroom 3 grams daily, and melatonin 20 mg. The patient was enrolled in a clinical trial, to receive NPDL, an inhibitor of programmed cell death ligand given intravenously once every three weeks. Three months following initiation of clinical trail, the patient's CT scan revealed enlarged lesions in the right chest wall, and a new pulmonary mass in the left upper lobe. Additionally, a single lytic bone lesion was found in the right rib. Due to disease progression, Avastin was added to his treatment plan.

Since the last office visit the patient reported doing well on the clinical trial and Avastin. CT of the chest, abdomen, and pelvis showed interval improvement with reduction of size with multiple metastatic foci. Integrative support included intravenous vitamins every other week, and previously described supplements and mushroom combination, Grifola frondosa, Ganoderma lucidum, Lentinus edodes 3 grams daily. The patient reported no notable side effects from the clinical trial, and hypertension was well managed. The patient stated that he felt great, similar to how he felt prior to his diagnosis, and denied any discomfort related to treatment. This patient is currently in his fifth year of continued integrative treatment, which includes persistent use of medicinal mushrooms.

\section{Results}

These cases highlight the integrative management of RCC and the improved survival time compared to standard treatment alone. Patients that present with localized disease can undergo curative surgical resection, but disease eventually recurs. Patients that have metastatic disease have a 5-year survival of $12.1 \%$ [1]. Prognostic factors in patients with advanced disease include performance status, serum lactic dehydrogenase levels, serum calcium, hemoglobin concentration, and absence of nephrectomy [34]. Patients with one or more factors present often have poorer survival rates at one year compared to patients without any risk factors. Both cases presented here had one or more risk factors at the time of diagnosis, suggestive of a poor prognosis and decreased overall survival. Despite the established risk factors, both patients maintained an improved quality of life and survived four and five years after diagnosis of RCC.

The standard adjuvant treatment is cytokine therapy and monoclonal antibodies, which offer a progression of disease free survival of 5.4 months [35]. Previous to targeted therapies treatment focused on cytokine therapy (IL-2, and IFNa) following surgery, which provided remission in $10 \%$ of cases and is associated with significant toxicity [36]. It is well understood that the removal of primary RCC tumors can evoke immune responses, and immunotherapy can provide remission in select patients, which ultimately suggests that RCC can respond to immunotherapies. An immunotherapy that offers an extended and robust immune response, survival beyond 5.4 
Citation: Costa M, Panutich E, Zwickey H (2016) Renal Cell Carcinoma: A Case Series with Integrative Treatment. J Integr Oncol S1: 002. doi:10.4172/2329-6771.S1-002

Page 4 of 5

\begin{tabular}{|c|c|c|c|c|c|}
\hline Scientific Name & Common Name & Immunomodulating Constituents & Structure of Immunomodulators & $\begin{array}{l}\text { Cytokines } \\
\text { Increased }\end{array}$ & Ref \\
\hline Ganoderma lucidum & $\begin{array}{l}\text { Reishi, } \\
\text { Ling Zhi }\end{array}$ & $\begin{array}{l}\text { Ganopoly, } \\
\text { Ganoderans }\end{array}$ & $\begin{array}{l}\text { Glycoproteins: } \\
\text { Heteropolysacch-arides } \\
\text { B-D-glucans }\end{array}$ & $\begin{array}{l}\text { IL-2, IFN-Y } \\
\text { NFkB }\end{array}$ & $37-40$ \\
\hline Grifola frondosa & Maitake & $\begin{array}{c}\text { MD-fraction, } \\
\text { Hydroxymethylacylf-ulvene }\end{array}$ & $\begin{array}{l}\text { Polysaccharides: } \\
\text { (1-6)-b-D-glucan } \\
\text { (1-3)-b-D side chains }\end{array}$ & $\begin{array}{l}\text { IFNy } \\
\text { IL-1 } \\
\text { IL-2 } \\
\text { IL-12 }\end{array}$ & $41-43$ \\
\hline Cordyceps sinensis & Caterpillar & Cordyceptin & Biomass nucleotides & $\begin{array}{l}\text { IL-1 } \\
\text { IL-2 } \\
\text { NFkB }\end{array}$ & 44 \\
\hline Lentinus edodes & Shiitake & Lentinans (LNT) & $\begin{array}{c}\text { Polysaccharides: } \\
\text { (1-3)-b-D-glucan with (1-6)-b-D-glucosyl branches }\end{array}$ & $\begin{array}{l}\text { TNFa } \\
\text { IL-2 }\end{array}$ & $45-47$ \\
\hline $\begin{array}{l}\text { Trametes versicolor/ } \\
\text { Coriolus versicolor }\end{array}$ & Turkey Tail & Krestin, PSK & $\begin{array}{l}\text { Polysaccharide peptides: } \\
\text { Heteroglucans with a (1-4)- and b-(1-3) glycosidic } \\
\text { linkages } \\
\text { Biomass }\end{array}$ & $\begin{array}{l}\text { TNFa } \\
\text { IFNy } \\
\text { IL-12 }\end{array}$ & 48 \\
\hline
\end{tabular}

Table 1: Medicinal Mushrooms \& Active Constituents.

months, and improves the quality of life in RCC patients is urgently needed. Medicinal mushrooms are hypothesized to contribute to these objectives in this case series.

The mushrooms previously described, Ganoderma lucidum, Trametes versicolor, Cordyceps sinensis, Grifola frondosa, Lentinus edodes, exhibit effective anticancer pharmacokinetics that prevent evasion of anti-growth signals, apoptosis, angiogenesis, and promote specific cytokine profiles important in modifying the immune response to cancer (Table 1, [37-48]). The polysaccharides from these mushrooms can promote a shift in the pattern of cytokines to a Th1 dominant, cytotoxic pattern, increasing IL-2 and IFN- $\partial$ while decreasing cytokines such as IL-4 [49]. These mushrooms can increase endogenous cytokine IL-2 without causing the toxicity found with exogenous administration of bolus levels found in immunotherapy for RCC (Table 1, [49, 50]).

\section{Conclusions}

Over the course of four and five years these patients experienced multiple surgeries and adjuvant therapies and integrative care. Case 1 described a patient that survived four years with a very poor prognosis due to metastatic disease to the bladder with a 3-year survival rate of $20 \%$ [12]. Integrative care for these cases included medicinal mushrooms dosed between 3-4 grams daily, and mushrooms were rotated and administered before, during, and after chemotherapy. Given the abundant evidence in the literature on the anti-cancer effects of mushrooms it is possible to speculate that the mushroom combinations prescribed in these cases contributed to the improved quality of life and the significant extended survival in these patients.

The NCCN guidelines suggest clinical trials for experimental drugs and radiation for cancer patients. Unfortunately, the currently available clinical trials for RCC do not include experimental combinations of medicinal mushrooms administered with standard of care. The pathophysiology of RCC and the mechanisms of intrinsic and acquired resistance to therapy are complex. A reductionist approach to therapy is clearly not offering clinical outcomes needed for RCC. Integrative medicine research that includes a whole systems approach with a focus on quality of life and extended survival in renal cell carcinoma should include the mentioned medicinal mushrooms to create new strategies in treatment.

\section{References}

1. http://seer.cancer.gov/statfacts/html/kidrp.html

2. Robbins, Cotran (2010) The Kidney: In: Pathological Basis of Disease (8thedn), W.B Saunders Company Philadelphia, USA.

3. Garnick MB (1998) Primary neoplasms of the kidney. Therapy in Nephrology and Hypertension: A Companion to Brenner and Rector's the Kidney. Philadelphia 337-340.

4. Bianchi M, Sun M, Jeldres C, Shariat SF, Trinh QD, et al. (2012) Distribution of metastatic sites in renal cell carcinoma: a population-based analysis.Ann Oncol 23: 973-980.

5. Atkins MB (2015) Overview of the treatment of renal cell carcinoma.

6. Russo $P$ (2010) Commentary on Urinary collecting system invasion is an independent prognostic factor of organ confined renal cell carcinoma. Urol Oncol 28: 228-229.

7. Anderson CB, Clark PE, Morgan TM, Stratton KL, Herrell SD, et al. (2011) Urinary collecting system invasion is a predictor for overall and disease-specific survival in locally invasive renal cell carcinoma. Urology 78: 99-104.

8. Cho HJ, Kim SJ, Ha US, Hong SH, Kim JC, et al. (2009) Prognostic value of capsular invasion for localized clear-cell renal cell carcinoma. Eur Urol, 56: 1006-1012.

9. Heng DYC, Choueiri TK, Rini BI, Lee J, Yuasa T, et al. (2014) Outcomes of patients with metastatic renal cell carcinoma that do not meet eligibility criteria for clinical trials. Ann Oncol 25: 149-154.

10. Waters JS, Moss C, Pyle L, James M, Hackett S, et al. (2004) Phase II clinical trial of capecitabine and gemcitabine chemotherapy in patients with metastatic renal carcinoma. Br J Cancer 91: 1763-1768.

11. George CM, Vogelzang NJ, Rini BI, Geoffroy FJ, Kollipara P, et al. (2002) A phase II trial of weekly intravenous gemcitabine and cisplatin with continuous infusion fluorouracil in patients with metastatic renal cell carcinoma. Ann Oncol 13: $116-120$.

12. Matsuo M, Koga S, Nishikido M, Noguchi M, Sakaguchi M, et al. (2002) Rena cell carcinoma with solitary metachronous metastasis to the urinary bladder. Urology 60: 911-912.

13. Kanzaki R, Higashiyama M, Fujiwara A, Tokunaga T, Maeda J, et al. (2011) Long-term results of surgical resection for pulmonary metastasis from renal cell carcinoma: a 25-year single-institution experience. Eur J Cardiothorac Surg 39: $167-172$.

14. Kaur G, Verma N (2015) Nature curing cancer - review on structural modification studies with natural active compounds having anti-tumor efficiency. Biotechnol Reports 6: 64-78.

15. Moradali MF, Mostafavi H, Ghods S, Hedjaroude GA (2007) Immunomodulating and anticancer agents in the realm of macromycetes fungi (macrofungi). Int Immunopharmacol 7: 701-724. 
Citation: Costa M, Panutich E, Zwickey H (2016) Renal Cell Carcinoma: A Case Series with Integrative Treatment. J Integr Oncol S1: 002. doi:10.4172/2329-6771.S1-002

Page 5 of 5

16. Roupas $P$, Keogh J, Noakes M, Margetts C Taylor P (2012) The role of edible mushrooms in health: Evaluation of the evidence. J Funct Foods 4: 687-709.

17. Vinay DS, Ryan EP, Pawelec G, Talib WH, Stagg J, et al. (2015) Immune evasion in cancer: Mechanistic basis and therapeutic strategies. Semin Cancer Biol 35 Suppl: S185-198.

18. Zong A, Cao H, Wang F (2012) Anticancer polysaccharides from natura resources: a review of recent research. Carbohydr Polym 90: 1395-1410.

19. Cao QZ, Lin ZB (2006) Ganoderma lucidum polysaccharides peptide inhibits the growth of vascular endothelial cell and the induction of VEGF in human lung cancer cell. Life Sci 78: 1457-1463.

20. Sadava D, Still DW, Mudry RR, Kane SE (2009) Effect of Ganoderma on drugsensitive and multidrug-resistant small-cell lung carcinoma cells. Cancer Lett 277: 182-189.

21. Lu QY, Jin YS, Zhang Q, Zhang Z, Heber D, et al. (2004) Ganoderma lucidum extracts inhibit growth and induce actin polymerization in bladder cancer cells in vitro. Cancer Lett 216: 9-20.

22. Eliza WL, Fai CK, Chung LP (2012) Efficacy of Yun Zhi (Coriolus versicolor) on survival in cancer patients: systematic review and meta-analysis. Recent Pat Inflamm Allergy Drug Discov 6: 78-87.

23. Hsieh T, Wu P, Park S, Wu JM (2006) Induction of cell cycle changes and modulation of apoptogenic/anti-apoptotic and extracellular signaling regulatory protein expression by water extracts of l'm-Yunity (PSP). BMC Complement Altern Med 6: 30

24. Ono Y, Hayashida T, Konagai A, Okazaki H, Miyao K, et al. (2012) Direct inhibition of the transforming growth factor- $\beta$ pathway by protein-bound polysaccharide through inactivation of Smad2 signaling. Cancer Sci 103: 317324

25. Masuda Y, Inoue M, Miyata A, Mizuno S, Nanba H (2009) Maitake beta-glucan enhances therapeutic effect and reduces myelosupression and nephrotoxicity of cisplatin in mice. Int Immunopharmacol 9: 620-626.

26. Cui FJ, Tao WY, Xu ZH, Guo WJ, Xu HY, et al.(2007) Structural analysis of anti-tumor heteropolysaccharide GFPS1b from the cultured mycelia of Grifola frondosa GF9801. Bioresour Technol 98: 395-401.

27. Klaus A, Kozarski M, Vunduk J, Todorovicb N, Jakovljevicb D, et al.(2015) Biological potential of extracts of the wild edible Basidiomycete mushroom Grifola frondosa. Food Res Int 67: 272-283.

28. Sun M, Zhao W, Xie Q, Zhan Y, Wu B (2015) Lentinan reduces tumor progression by enhancing gemcitabine chemotherapy in urothelial bladde cancer. Surg Oncol 24: 28-34.

29. Jeff IB, Yuan X, Sun L, Kassim RMR, Foday AD, et al.(2013) Purification and in vitro anti-proliferative effect of novel neutral polysaccharides from Lentinus edodes. Int J Biol Macromol 52: 99-106.

30. Chen PX, Wang S, Nie S, Marcone M (2013) Properties of Cordyceps Sinensis: A review. J Funct Foods 5: 550-569.

31. Nakamura K, Shinozuka K, Yoshikawa N (2015) Anticancer and antimetastatic effects of cordycepin, an active component of Cordyceps sinensis. J Pharmacol Sci 127: 53-56.

32. Cheng JH, Guo XM, Wang X (1995) Analysis of therapeutic effects of Jinshuibao capsule in adjuvant treatment of 20 patients with terminal stage of lung cancer. J Admin Tradit Chin Med 5: 34-35

33. Yan RJ, Li YZ, Su RX (1992) Observation of efficacy of XinGan-Bao capsule and other Cordyceps mycelia preparations as a main therapy on treating 50 patients with lung cancer. Chinese J Hospital Therapeutics 12: 61-63.

34. Choueiri TK (2015) Prognostic factors in patients with renal cell carcinoma UpToDate.
35. Escudier B, Pluzanska A, Koralewski P, Ravaud A, Bracarda S, et al. (2007) Bevacizumab plus interferon alfa-2a for treatment of metastatic renal cel carcinoma: arandomised, double-blind phase III trial. Lancet 370: 2103-2111.

36. George D, Jonasch E (2015) Immunotherapy of renal cell carcinoma UpToDate.

37. Wang G, Zhao J, Liu J, Huang Y, Zhong JJ, et al. (2007) Enhancement of IL-2 and IFN-gamma expression and NK cells activity involved in the anti-tumor effect of ganoderic acid Me in vivo. International Immunopharmacology 7: 864870

38. Yuen J, Gohel M, Ng C (2011) The differential immunological activities of Ganoderma lucidum on human pre-cancerous uroepithelial cells. Journal of ethnopharmacology 135: 711-718.

39. Gao Y, Zhou S, Jiang W, Huang M, Dai X (2003) Effects of ganopoly (a Ganoderma lucidum polysaccharide extract) on the immune functions in advanced-stage cancer patients. Immunological Investigations 32: 201-215.

40. Gao Y, Tang W, Gao H, Chan E, Jin Lan, et al. (2005) Antimicrobial activity of the medicinal mushroom Ganoderma. Food Reviews International 21: 211-229.

41. Kodama N, Harada N, Nanba $\mathrm{H}$ (2002) A polysaccharide, extract from Grifola frondosa, induces Th-1 dominant responses in carcinomabearing BALB/c mice. The Japanese Journal of Pharmacology 90: 357-360.

42. Masuda Y, Murata Y, Hayashi M, Nanba H (2008) Inhibitory effect of MD Fraction on tumor metastasis: involvement of NK cell activation and suppression of intercellular adhesion molecule (ICAM)-1 expression in lung vascular endothelial cells. Biological and Pharmaceutical Bulletin 31: 1104-1108.

43. Mayell M (2001) Maitake extracts and their therapeutic potential. Altern Med Rev 6: 48-60.

44. Kawanishi T, Ikeda-Dantsuji Y, Nagayama A (2010) Effects of two basidiomycete species on interleukin 1 and interleukin 2 production by macrophage and T cell lines. Immunobiology 215: 516-520.

45. Zheng R, Jie S, Hanchuan D, Moucheng W (2005) Characterization and immunomodulating activities of polysaccharide from Lentinus edodes. Int Immunopharmacol 5: 811-820.

46. Liu M, Li J, Kong F, Lin J, Gao Y (1998) Induction of immunomodulating cytokines by a new polysaccharide-peptide complex from culture mycelia of Lentinus edodes. Immunopharmacology 40: 187-198.

47. Lu H, Yang Y, Gad E, Wnner CA, Chang A, et al.(2011) Polysaccharide krestin is a novel TLR2 agonist that mediates inhibition of tumor growth via stimulation of CD8 T cells and NK cells. Clinical cancer research 17: 67-76.

48. Powell M (2010) Medicinal Mushrooms A Clinical Guide. United Kingdom.

49. Lu H, Yang Y, Gad E, Inatsuka C, Wenner CA, et al. (2011) TLR2 agonist PSK activates human NK cells and enhances the antitumor effect of HER2-targeted monoclonal antibody therapy. Clin Cancer Res 17: 6742-6753.

50. Wang PY, Zhu XL, Lin ZB (2012) Antitumor and Immunomodulatory Effects of Polysaccharides from Broken-Spore of Ganoderma lucidum. Front Pharmacol 3: 135 